\title{
Research Article \\ Pilot Study of Acupuncture Point Laterality: Evidence from Heart Rate Variability
}

\author{
Guangjun Wang, Yuying Tian, Shuyong Jia, Wenting Zhou, and Weibo Zhang \\ Institute of Acupuncture and Moxibustion, China Academy of Chinese Medical Sciences, 16 Dongzhimennei, Nanxiaojie, \\ Dongchen District, Beijing 100700, China
}

Correspondence should be addressed to Weibo Zhang; zhangweibo@hotmail.com

Received 16 September 2013; Revised 6 November 2013; Accepted 6 November 2013

Academic Editor: Gerhard Litscher

Copyright (C) 2013 Guangjun Wang et al. This is an open access article distributed under the Creative Commons Attribution License, which permits unrestricted use, distribution, and reproduction in any medium, provided the original work is properly cited.

The specificity of acupuncture points (acupoints) is one of the key concepts in traditional acupuncture theory, but the question of whether there is adequate scientific evidence to prove or disprove specificity has been vigorously debated in recent years. Laterality, or the tendency for acupoints on the right or left side of the body to produce different physiological effects, is an important aspect of acupoint specificity. Data is particularly scarce regarding the laterality of the same channel, same-named acupoint located on opposite sides of the body. The aim of this study was to investigate whether Neiguan (PC6) has laterality. A total of eighteen healthy female volunteers were recruited for this study. Electrocardiograms were recorded and heart rate variability was analyzed before, during, and after PC6 was stimulated on either the left or the right side. The results show that during acupuncture, there were significant differences in the standard deviation of RR intervals (STDRR), root mean square of successive differences between RR intervals (RMSSD), and total power between the left PC6 stimulation group and the right PC6 stimulation group, which indicates that PC6 may have laterality.

\section{Introduction}

Acupuncture has been widely used as a healing modality for at least 2500 years [1]. Over the past 30 years, researchers have demonstrated the neurobiological basis for the analgesic effects of acupuncture, which has led to greater acceptance of acupuncture in the scientific community $[2,3]$. However, a number of well-designed clinical trials have reported that although "true" acupuncture is superior to usual care, it does not significantly outperform sham acupuncture $[1,4]$. These findings are apparently at odds with traditional theories regarding acupuncture point specificity because in traditional Chinese medicine (TCM) theory, acupoint specificity is an essential principle for therapeutic efficacy. On the other hand, some clinical trials $[5,6]$ and studies using fMRI technology $[7,8]$ have demonstrated acupoint specificity.

For example, in a previous study performed by our group, we found that ipsilateral stimulation of Hegu (LI4) corresponded to increased blood perfusion in the contralateral Hegu (LI4) [9]. Moreover, the increased degree of blood perfusion was asymmetrical [10], which suggests the laterality, or specificity, of this acupuncture point [11]. In clinical practice, Neiguan (PC6) is one of the most commonly used acupoints and is indicated for treating cardiovascular related disorders in classical texts [12-14]. However, the differences between the bilateral PC6 acupoints have never been scientifically investigated. The aim of this study is to investigate the specificity of bilateral PC6 points based on heart rate variability.

\section{Materials and Methods}

2.1. Ethics Statement. This study was reviewed and approved by the Institutional Review Board at the Institute of Acupuncture and Moxibustion, China Academy of Chinese Medical Sciences. Each participant read and signed an informed consent form.

2.2. Subjects. 18 healthy female volunteers were recruited in this study. All subjects were students from the China Academy of Chinese Medical Sciences and Beijing University of TCM. None of the subjects had a history of prior disease 


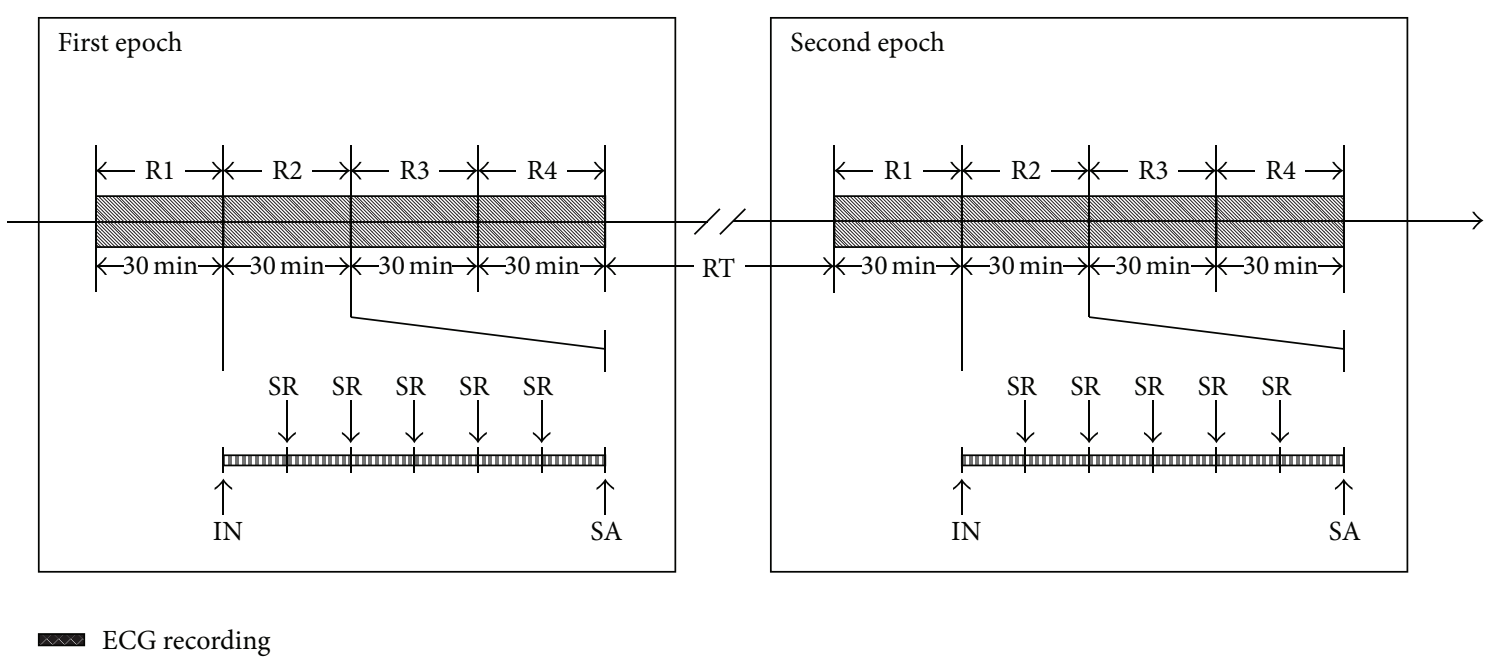

FIGURE 1: Procedure of acupuncture and measurement. $R_{i}(i=1,2,3,4)$ : ECG recording time point; IN: insert needle; SA: stop acupuncture; SR: slowly rotate the needle every $5 \mathrm{~min}$ (the needle was slowly rotated every $5 \mathrm{~min}$ for a total of $30 \mathrm{~min}$ during the R2 acupuncture session); RT: rest time between two epochs, the range is $8-22$ days.

TABLE 1: Basic characteristics of the study participants $(n=18)$.

\begin{tabular}{lcc}
\hline Characters & Mean \pm SD & Range \\
\hline Age (years) & $25.61 \pm 2.38$ & $33-21$ \\
High $(\mathrm{cm})$ & $161.78 \pm 4.60$ & $172-150$ \\
Body weight $(\mathrm{kg})$ & $50.50 \pm 4.89$ & $60-42$ \\
Body mass index (BMI) & $19.28 \pm 1.58$ & $22.04-15.79$ \\
Interval of two measures (days) & $13.28 \pm 3.99$ & $22-8$ \\
\hline
\end{tabular}

nor had they taken any medication in the six months prior to the study. Each subject was provided with informed consent and had an adequate understanding of the procedure and purpose of this study. Basic characteristics of the participants are shown in Table 1 .

2.3. Electrocardiogram Measurement Protocol. Before the laboratory procedure began, subjects were placed in a temperature-controlled room $\left(24-26^{\circ} \mathrm{C}\right)$ to rest for 10 minutes. The ECG recordings were processed with standard II electrocardiographic lead on NeurOne system (NeurOne, MEGA Electronics Ltd, Finland). The data were digitized with a sampling rate of $500 \mathrm{~Hz}$. In every epoch, the 4 segments of successive ECG were recorded and symbolized as R1 to R4. In each segment, a 30 min ECG recording was obtained using the NeurOne system (shown in Figure 1).

2.4. Acupuncture Protocol. For every participant, either the right or left PC6 was stimulated during the first epoch of the study and the opposite side PC6 was stimulated during the second epoch. The stimulus order was determined randomly and the interval time between the two epochs was at least 8 days. For the acupuncture procedure, a small acupuncture needle, $0.25 \times 25 \mathrm{~mm}$ (100112, Zhen Huan), was gently inserted to a depth of $15 \mathrm{~mm}$ in Neiguan (PC6). The needle was slowly rotated every $5 \mathrm{~min}$ for a total of $30 \mathrm{~min}$ during the acupuncture session in order to maintain the soreness and numbness sensation of De-Qic $[9,15]$. The acupuncture process is illustrated in Figure 1.

2.5. Data Analysis. The raw data recorded by NeurOne system was exported with ASC format and then imported into Kubio HRV software and analyzed [16]. The analysis parameter was default. In the time domain, the mean heart rate (HR), the standard deviation of RR intervals (STDRR), and the root mean square of successive differences (RMSSD) were analyzed. In the frequency domain, the power spectrum density was analyzed with AR spectrum method in normalized units. The low frequency (LF) and high frequency (HF) were defined as $0.04-0.15 \mathrm{~Hz}$ and $0.15-0.4 \mathrm{~Hz}$, respectively. Data are expressed as Mean $\pm \mathrm{SD}$. For every recording point, the paired $t$-test was performed between RS group and LS group. The level of significance was defined as $P<0.05$. Statistical analyses were performed using SPSS (SPSS Inc., Chicago, IL, USA).

\section{Results}

Table 1 and Figure 2(a) present the mean value of heart rate during the two epochs of the experiment. HR did not change significantly between the right side (RS) group and left side (LS) group.

3.1. Time Domain Results. Table 2 and Figures 2(b) and 2(c) present the results of time domain analysis during the preacupuncture, acupuncture, and postacupuncture periods. The results show that before and after acupuncture (R1, R3, and R4), there were no significant differences in STDRR and RMSSD between the LR-group and RS group. However, 


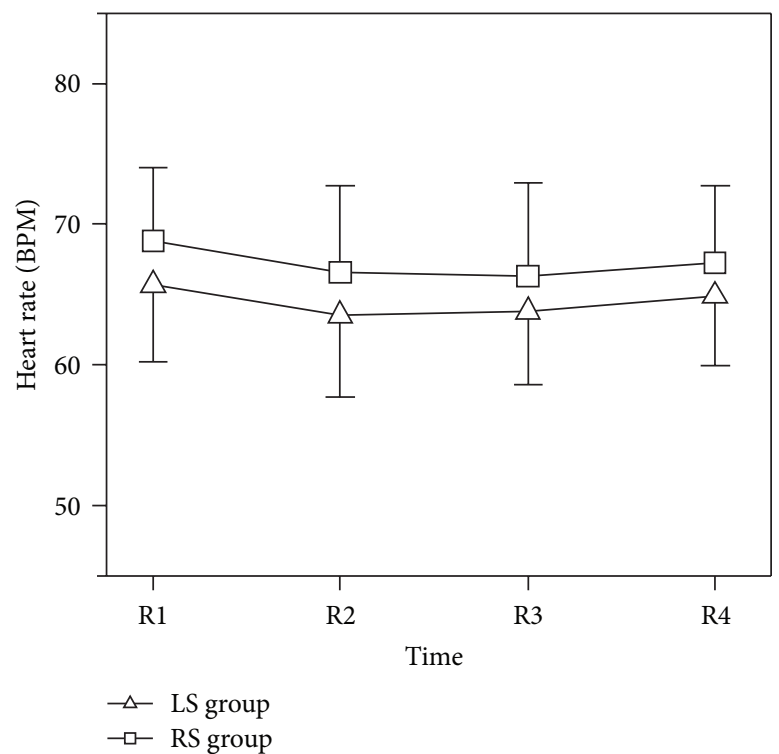

(a)

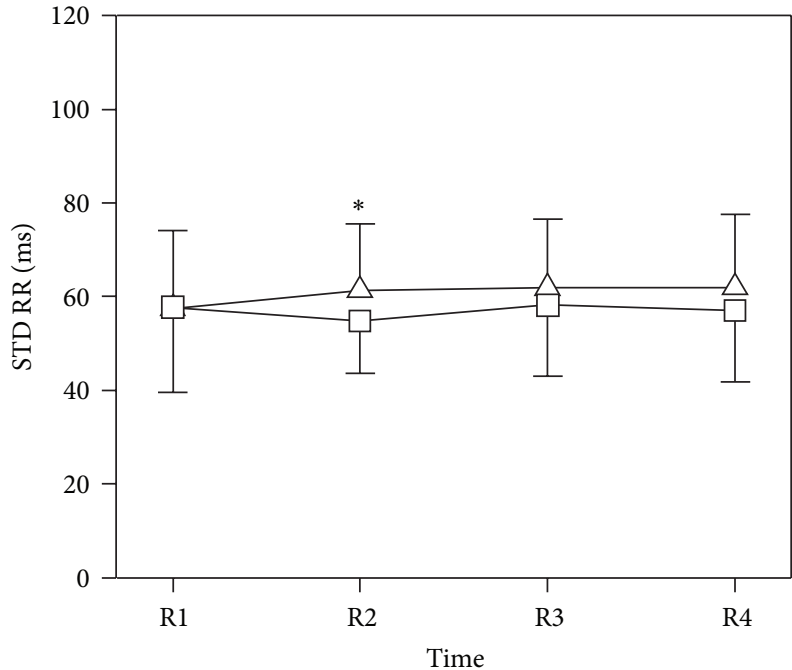

$\triangle-$ LS group

$\rightarrow-$ RS group

(b)

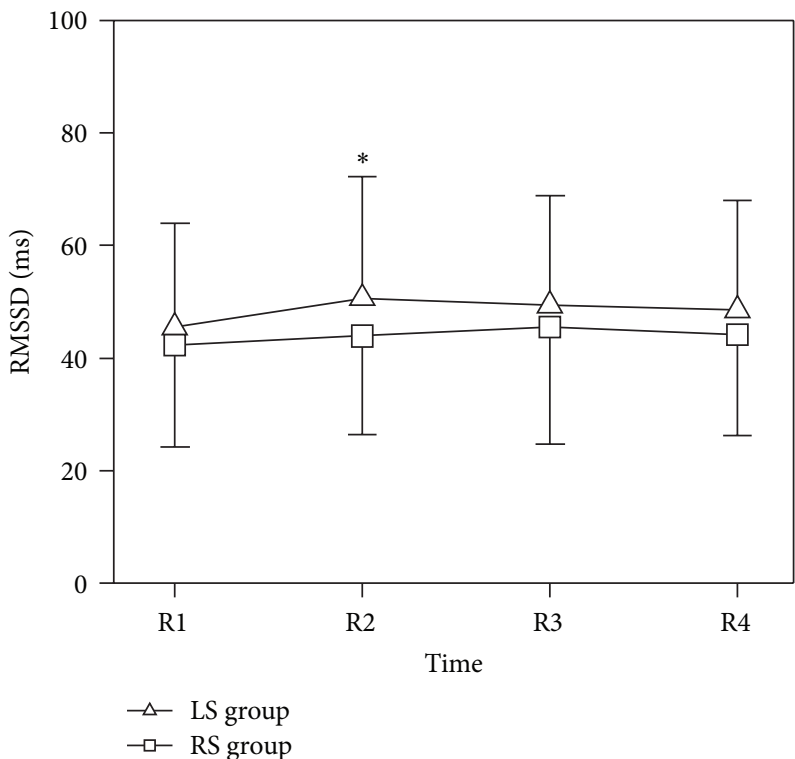

(c)

FIGURE 2: Time domain results. LS: left Neiguan acupoint was stimulated; RS: right Neiguan acupoint was stimulated; STDRR: standard deviation of RR intervals; RMSSD: root mean square of successive differences between RR intervals. Data are expressed as Mean \pm SD; ${ }^{*} P<0.05$; LS group versus RS group; one-sample paired $t$-test.

during the acupuncture, there were significant differences between the two groups.

3.2. Frequency Domain Results. Table 3 and Figure 3 present the results of frequency domain analysis during the preacupuncture, acupuncture, and postacupuncture periods. The results show that before, during, and after acupuncture (R1, R2, R3, and R4), there was no significant difference in LF, HF, or LF/HF ratio between the LR-group and RS group.
However, during the acupuncture, there were significant differences in total power between the two groups.

\section{Discussion}

When a needle is inserted into a point on the body, various neural and neuroactive components are activated $[17,18]$. Acupuncture has been shown to have clear central nervous 
TABLE 2: Time domain result of heart rate variability.

\begin{tabular}{|c|c|c|c|c|c|}
\hline & Time & LS group & RS group & $T$ value & $P$ value \\
\hline \multirow{4}{*}{ Mean HR (BPM) } & $\mathrm{R} 1$ & $65.669 \pm 5.439$ & $68.801 \pm 5.237$ & -1.914 & 0.073 \\
\hline & $\mathrm{R} 2$ & $63.505 \pm 5.771$ & $66.561 \pm 6.131$ & -1.886 & 0.077 \\
\hline & $\mathrm{R} 3$ & $63.752 \pm 5.145$ & $66.314 \pm 6.581$ & -1.581 & 0.132 \\
\hline & $\mathrm{R} 4$ & $64.897 \pm 4.970$ & $67.240 \pm 5.457$ & -1.641 & 0.119 \\
\hline \multirow{4}{*}{ STDRR (ms) } & $\mathrm{R} 1$ & $57.495 \pm 16.635$ & $57.737 \pm 18.111$ & -0.046 & 0.964 \\
\hline & $\mathrm{R} 2$ & $61.386 \pm 14.168$ & $54.763 \pm 11.235$ & 2.238 & 0.039 \\
\hline & $\mathrm{R} 3$ & $61.886 \pm 14.773$ & $58.182 \pm 15.163$ & 0.891 & 0.386 \\
\hline & $\mathrm{R} 4$ & $62.010 \pm 15.453$ & $57.088 \pm 15.399$ & 1.748 & 0.099 \\
\hline \multirow{4}{*}{ RMSSD (ms) } & $\mathrm{R} 1$ & $45.418 \pm 18.573$ & $42.256 \pm 18.084$ & 0.993 & 0.335 \\
\hline & $\mathrm{R} 2$ & $50.629 \pm 21.593$ & $43.886 \pm 17.490$ & 2.492 & 0.023 \\
\hline & R3 & $49.330 \pm 19.469$ & $45.540 \pm 20.906$ & 1.122 & 0.277 \\
\hline & $\mathrm{R} 4$ & $48.488 \pm 19.496$ & $44.151 \pm 17.963$ & 1.714 & 0.105 \\
\hline
\end{tabular}

LS: left Neiguan acupoint was stimulated; RS: right Neiguan acupoint was stimulated; HR: heart rate; STDRR: standard deviation of RR intervals; RMSSD: root mean square of successive differences between RR intervals; BPM: beat per minute. Data are expressed as Mean \pm SD.

TABLE 3: Frequency domain result of heart rate variability.

\begin{tabular}{|c|c|c|c|c|c|}
\hline & Time & LS group & RS group & $T$ value & $P$ value \\
\hline \multirow{4}{*}{ LF } & $\mathrm{R} 1$ & $44.308 \pm 13.443$ & $47.128 \pm 16.006$ & -1.325 & 0.203 \\
\hline & $\mathrm{R} 2$ & $44.94 \pm 14.216$ & $48.373 \pm 16.697$ & -1.429 & 0.171 \\
\hline & $\mathrm{R} 3$ & $45.693 \pm 14.727$ & $48.755 \pm 19.521$ & -0.927 & 0.367 \\
\hline & $\mathrm{R} 4$ & $46.747 \pm 15.164$ & $50.605 \pm 15.892$ & -1.605 & 0.127 \\
\hline \multirow{4}{*}{$\mathrm{HF}$} & $\mathrm{R} 1$ & $55.618 \pm 13.433$ & $52.806 \pm 15.981$ & 1.322 & 0.204 \\
\hline & $\mathrm{R} 2$ & $54.988 \pm 14.217$ & $51.548 \pm 16.68$ & 1.432 & 0.170 \\
\hline & $\mathrm{R} 3$ & $54.245 \pm 14.728$ & $51.182 \pm 19.511$ & 0.927 & 0.367 \\
\hline & $\mathrm{R} 4$ & $53.184 \pm 15.169$ & $49.332 \pm 15.889$ & 1.604 & 0.127 \\
\hline \multirow{4}{*}{ Total Power } & $\mathrm{R} 1$ & $3258.025 \pm 1758.083$ & $3397.377 \pm 2253.850$ & -0.218 & 0.830 \\
\hline & $\mathrm{R} 2$ & $3613.889 \pm 1537.027$ & $2892.476 \pm 1143.268$ & 2.375 & 0.030 \\
\hline & $\mathrm{R} 3$ & $3738.289 \pm 1736.069$ & $3374.885 \pm 1663.170$ & 0.781 & 0.445 \\
\hline & $\mathrm{R} 4$ & $3761.157 \pm 1960.836$ & $3241.175 \pm 1700.276$ & 1.408 & 0.177 \\
\hline \multirow{4}{*}{$\mathrm{LF} / \mathrm{HF}$} & $\mathrm{R} 1$ & $0.912 \pm 0.517$ & $1.087 \pm 0.713$ & -1.634 & 1.121 \\
\hline & $\mathrm{R} 2$ & $0.928 \pm 0.463$ & $1.142 \pm 0.693$ & -2.055 & 0.056 \\
\hline & $\mathrm{R} 3$ & $0.979 \pm 0.547$ & $1.271 \pm 0.928$ & -1.855 & 0.081 \\
\hline & $\mathrm{R} 4$ & $1.052 \pm 0.672$ & $1.247 \pm 0.743$ & -1.627 & 0.122 \\
\hline
\end{tabular}

LF: low frequency; HF: high frequency; n.u.: normalized unit; LF/HF: low frequency high frequency ratio. Data are expressed as Mean \pm SD.

system and autonomic nervous system effects both in humans $[19,20]$ and in animals [21]. Previous studies showed that manual stimulation of Hegu (LI4) resulted in specific changes in alpha EEG frequency and in HRV parameters. The relationship between the HRV parameters and the special EEG band might point to a specific modulation of cerebral function by acupuncture [22]. On the other hand, power spectral analysis of heart rate variability (HRV) has recently been used as a sensitive index of autonomic nervous system activity. The analysis of HRV provides quantitative information regarding autonomic control mechanisms in the body [23]. For these reasons, HRV has recently been adopted as an index used to evaluate the effects of acupuncture [24].

A previous study indicated that the cardiac modulatory balance differs between genders and is characterized by a greater influence of the autonomic vagal component in women and by the sympathetic component in men [25].
Another study investigated the influence of age and gender on the short-term HRV indices and revealed significant modifications of the indices especially by age but partly also by gender, especially in the younger groups [26]. To exclude gender bias, we only recruited the healthy adult females in this study.

According to the traditional acupuncture theory, acupoints are distributed along meridians and will often have different effects in treatment. PC6 is a classic acupuncture point, and it is considered to be effective in treating cardiovascular disorders. Evidence has recently shown that violet laser stimulation at the PC6 induces a significant increase of total heart rate variability [27].

Previous studies demonstrated that acupuncture manipulation significantly decreased the LF spectral component of HRV and significantly reduced LF/HF, which is an index of sympathetic activity [28]. In the present study, acupuncture 


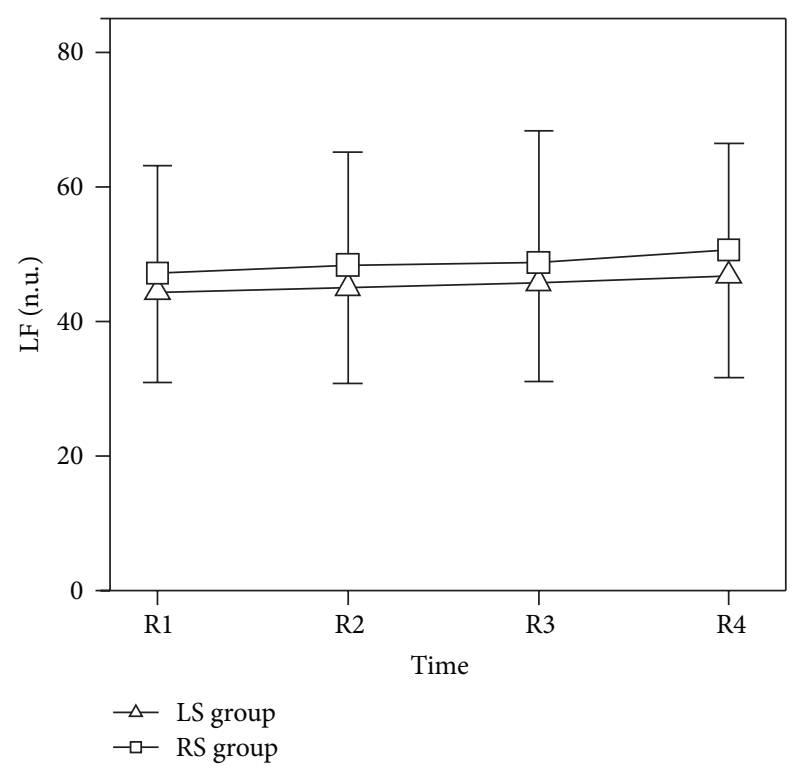

(a)

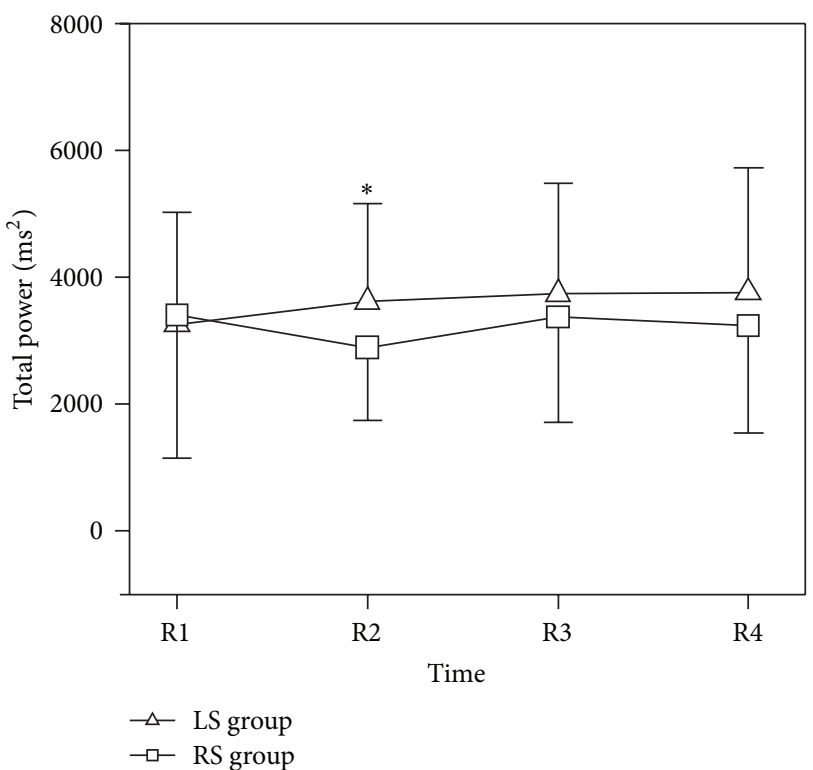

(c)

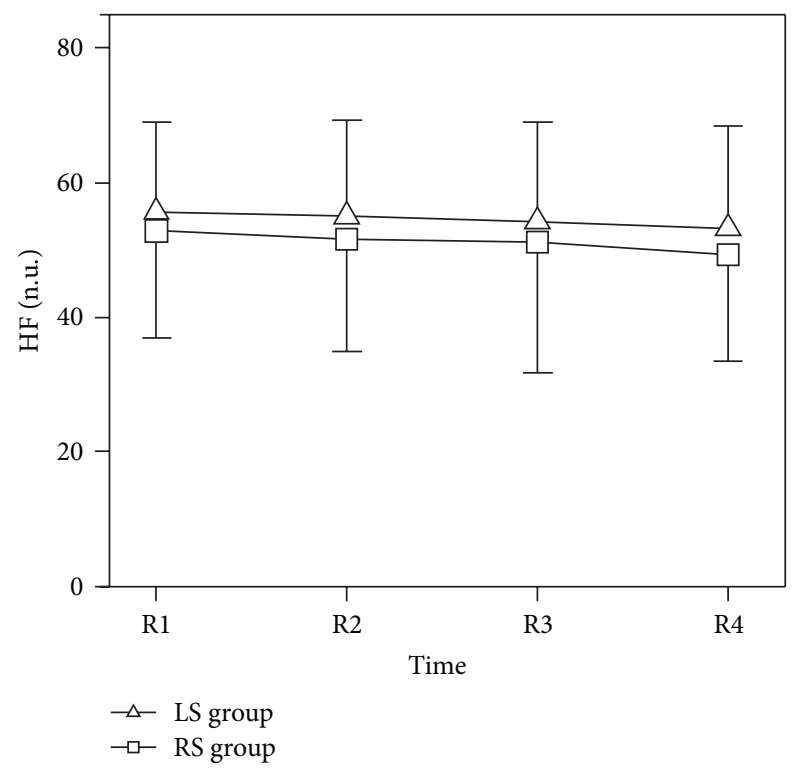

(b)

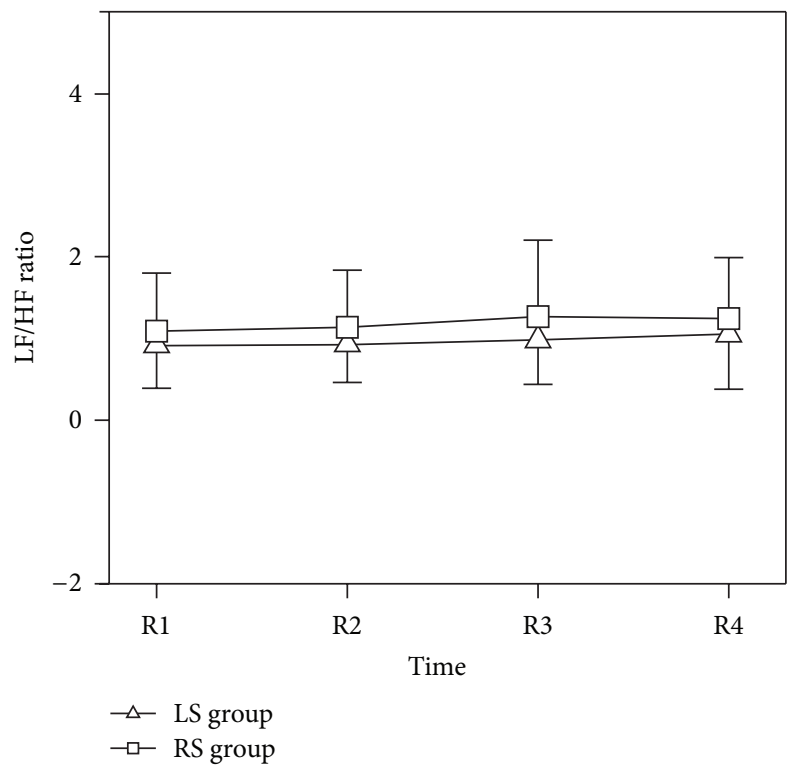

(d)

FIGURE 3: Frequency domain results. LS: left Neiguan acupoint was stimulated; RS: right Neiguan acupoint was stimulated; LF: low frequency; HF: high frequency; n.u.: normalized unit; LF/HF: low frequency high frequency ratio; PSD: Power spectrum density. Data are expressed as Mean $\pm \mathrm{SD} ;{ }^{*} P<0.05$; LS group versus RS group; one-sample paired $t$-test.

effects on heart rate variability mainly occurred in the acupuncture period. After the acupuncture was discontinued, this effect disappeared. Since the effects of acupuncture are the result of central nervous system regulation, we can expect laterality due to the fact that information is processed in the brain, which has hemispheric dominance [29]. In this study, differences in LF, HF, and LF/HF between the two groups were not observed; however, the difference in total power was significant.

Obviously, this is a plot study in acupoint laterality, the conclusions just resulted from the female healthy subjects aged from 21 to 33, and just the PC6 was investigated in the study. So, we cannot be sure whether all acupoints have the laterality, and we also cannot be sure whether this laterality will be changed under different conditions such as disorder, handedness, or aging. In particular, we note that the laterality of PC6 was just based on the HRV analysis. The mechanism and factors analysis needed further research.

\section{Acknowledgments}

This research was supported by the National Natural Science Foundation of China (81001553) and the Fundamental 
Research Funds for the Central Public Welfare Research Institutes (ZZ070806).

\section{References}

[1] H. M. Langevin, P. M. Wayne, H. MacPherson et al., "Paradoxes in acupuncture research: strategies for moving forward," Evidence-Based Complementary and Alternative Medicine, vol. 2011, Article ID 180805, 11 pages, 2011.

[2] T. A. McNearney, H. S. Sallam, S. E. Hunnicutt, D. Doshi, and J. D. Chen, "Prolonged treatment with transcutaneous electrical nerve stimulation (TENS) modulates neuro-gastric motility and plasma levels of vasoactive intestinal peptide (VIP), motilin and interleukin-6 (IL-6) in systemic sclerosis," Clinical and Experimental Rheumatology, vol. 31, no. 2, supplement 76, pp. 140-150, 2013.

[3] W. He, D. Wedig, L. Wang, I. Gaischek, and G. Litscher, "Violet laser acupuncture-part 5: an investigation of different stimulation frequencies on heart rate and variability," Journal of Acupuncture and Meridian Studies, vol. 5, no. 6, pp. 290-294, 2012.

[4] M. V. Madsen, P. C. Gøtzsche, and A. Hróbjartsson, "Acupuncture treatment for pain: systematic review of randomised clinical trials with acupuncture, placebo acupuncture, and no acupuncture groups," The British Medical Journal, vol. 338, Article ID a3115, 2009.

[5] Y. Li, H. Zheng, C. M. Witt et al., "Acupuncture for migraine prophylaxis: a randomized controlled trial," Canadian Medical Association Journal, vol. 184, no. 4, pp. 401-410, 2012.

[6] T. T. Ma, S. Y. Yu, Y. Li et al., "Randomised clinical trial: an assessment of acupuncture on specific meridian or specific acupoint versus sham acupuncture for treating functional dyspepsia," Alimentary Pharmacology and Therapeutics, vol. 35, no. 5, pp. 552-561, 2012.

[7] J. L. Fang, X. L. Wang, Y. Wang et al., "Electro-acupuncture at different acupoints modulating the relative specific brain functional network," in 4th Optics in Health Care and Biomedical Optics, vol. 7845 of Proceedings of SPIE, Beijing, China, October 2010.

[8] L. J. Bai, H. Yan, L. L. Li et al., "Neural specificity of acupuncture stimulation at pericardium 6: evidence from an fMRI study," Journal of Magnetic Resonance Imaging, vol. 31, no. 1, pp. 71-77, 2010.

[9] W. Guangjun, T. Yuying, J. Shuyong, H. Tao, and Z. Weibo, "Change of blood perfusion in Hegu acupoint after contralateral Hegu acupoint was stimulated," Journal of Alternative and Complementary Medicine, vol. 18, no. 8, pp. 784-788, 2012.

[10] G. Wang, J. Han, G. Litscher, and W. Zhang, "System identification algorithm analysis of acupuncture effect on mean blood flux of contralateral Hegu acupoint," Evidence-Based Complementary and Alternative Medicine, vol. 2012, Article ID 951928, 7 pages, 2012.

[11] G. J. Wang, "Study on acupoint laterality: the important supplement to acupoint specificity," Zhongguo Zhen Jiu, vol. 32, pp. 709-712, 2012.

[12] Y. C. P. Arai, N. Kato, M. Matsura et al., "Transcutaneous electrical nerve stimulation at the PC-5 and PC- 6 acupoints reduced the severity of hypotension after spinal anaesthesia in patients undergoing Caesarean section," The British Journal of Anaesthesia, vol. 100, no. 1, pp. 78-81, 2008.

[13] C. M. Witt, K. Meissner, D. Pach et al., "Stimulation of gastric slow waves with manual acupuncture at acupuncture points
ST36 and PC6-a randomized single blind controlled trial," Neurogastroenterology and Motility, vol. 24, no. 5, pp. 438-445, 2012.

[14] J. H. Lin, C. H. Shih, K. Kaphle et al., "Acupuncture effects on cardiac functions measured by cardiac magnetic resonance imaging in a feline model," Evidence-Based Complementary and Alternative Medicine, vol. 7, no. 2, pp. 169-176, 2010.

[15] N. Goldman, M. Chen, T. Fujita et al., "Adenosine A1 receptors mediate local anti-nociceptive effects of acupuncture," Nature Neuroscience, vol. 13, no. 7, pp. 883-888, 2010.

[16] J. Niskanen, M. P. Tarvainen, P. O. Ranta-Aho, and P. A. Karjalainen, "Software for advanced HRV analysis," Computer Methods and Programs in Biomedicine, vol. 76, no. 1, pp. 73-81, 2004.

[17] Z. J. Zhang, X. M. Wang, and G. M. McAlonan, "Neural acupuncture unit: a new concept for interpreting effects and mechanisms of acupuncture," Evidence-Based Complementary and Alternative Medicine, vol. 2012, Article ID 429412, 23 pages, 2012.

[18] M. H. Kim, Y. C. Park, and U. Namgung, "Acupuncturestimulated activation of sensory neurons," Journal of Acupuncture and Meridian Studies, vol. 5, no. 4, pp. 148-155, 2012.

[19] K. Nishijo, H. Mori, K. Yosikawa, and K. Yazawa, "Decreased heart rate by acupuncture stimulation in humans via facilitation of cardiac vagal activity and suppression of cardiac sympathetic nerve," Neuroscience Letters, vol. 227, no. 3, pp. 165-168, 1997.

[20] J. D. Wang, T. B. J. Kuo, and C. C. H. Yang, "An alternative method to enhance vagal activities and suppress sympathetic activities in humans," Autonomic Neuroscience, vol. 100, no. 1-2, pp. 90-95, 2002.

[21] X. Y. Gao, S. P. Zhang, B. Zhu, and H. Q. Zhang, "Investigation of specificity of auricular acupuncture points in regulation of autonomic function in anesthetized rats," Autonomic Neuroscience, vol. 138, no. 1-2, pp. 50-56, 2008.

[22] K. Streitberger, J. Steppan, C. Maier, H. Hill, J. Backs, and K. Plaschke, "Effects of verum acupuncture compared to placebo acupuncture on quantitative EEG and heart rate variability in healthy volunteers," Journal of Alternative and Complementary Medicine, vol. 14, no. 5, pp. 505-513, 2008.

[23] "Heart rate variability: standards of measurement, physiological interpretation and clinical use. Task force of the European society of cardiology and the North American society of pacing and electrophysiology," Circulation, vol. 93, no. 5, pp. 1043-1065, 1996.

[24] Z. Y. Li, C. T. Wang, A. F. T. Mak, and D. H. K. Chow, "Effects of acupuncture on heart rate variability in normal subjects under fatigue and non-fatigue state," European Journal of Applied Physiology, vol. 94, no. 5-6, pp. 633-640, 2005.

[25] S. G. Dutra, A. P. Pereira, G. C. Tezini, J. H. Mazon, M. C. Martins-Pinge, and H. C. D. Souza, "Cardiac autonomic modulation is determined by gender and is independent of aerobic physical capacity in healthy subjects," PLoS ONE, vol. 8, no. 10, Article ID e77092, 2013.

[26] A. Voss, R. Schroeder, C. Fischer, A. Heitmann, A. Peters, and S. Perz, "Influence of age and gender on complexity measures for short term heart rate variability analysis in healthy subjects," in Proceedings of the 35th Annual International Conference of the IEEE Engineering in Medicine and Biology Society (EMBC '13), pp. 5574-5577, Osaka, Japan, July 2013.

[27] G. Litscher, L. Wang, X. Wang, and I. Gaischek, "Laser acupuncture: two acupoints (Baihui, Neiguan) and two modalities 
of laser $(658 \mathrm{~nm}, 405 \mathrm{~nm})$ induce different effects in neurovegetative parameters," Evidence-Based Complementary and Alternative Medicine, vol. 2013, Article ID 432764, 6 pages, 2013.

[28] S. Sakai, E. Hori, K. Umeno, N. Kitabayashi, T. Ono, and H. Nishijo, "Specific acupuncture sensation correlates with EEGs and autonomic changes in human subjects," Autonomic Neuroscience, vol. 133, no. 2, pp. 158-169, 2007.

[29] J. L. van Hemmen and T. J. Sejnowski, 23 Problems in Systems Neuroscience, Oxford University Press, New York, NY, USA, 2006. 


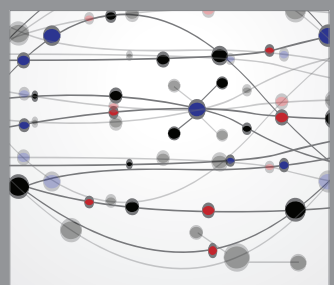

The Scientific World Journal
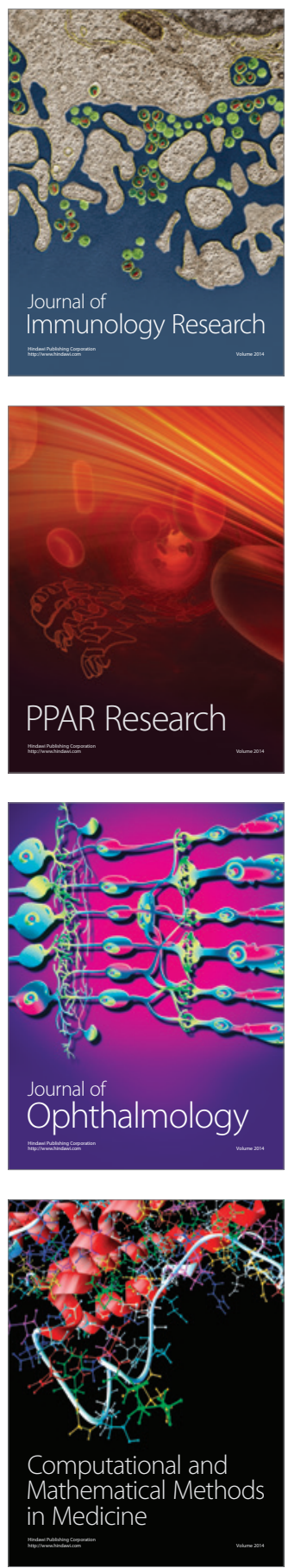

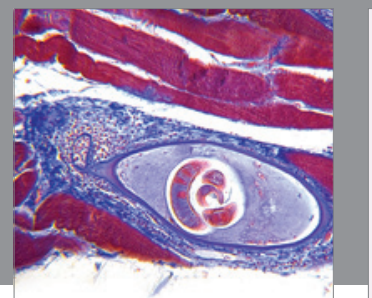

Gastroenterology

Research and Practice
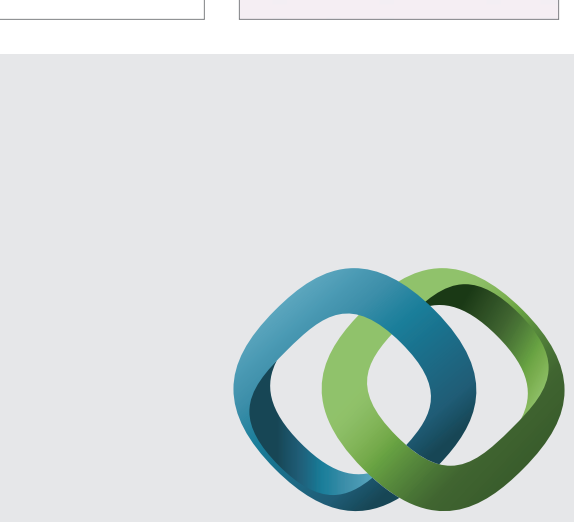

\section{Hindawi}

Submit your manuscripts at

http://www.hindawi.com
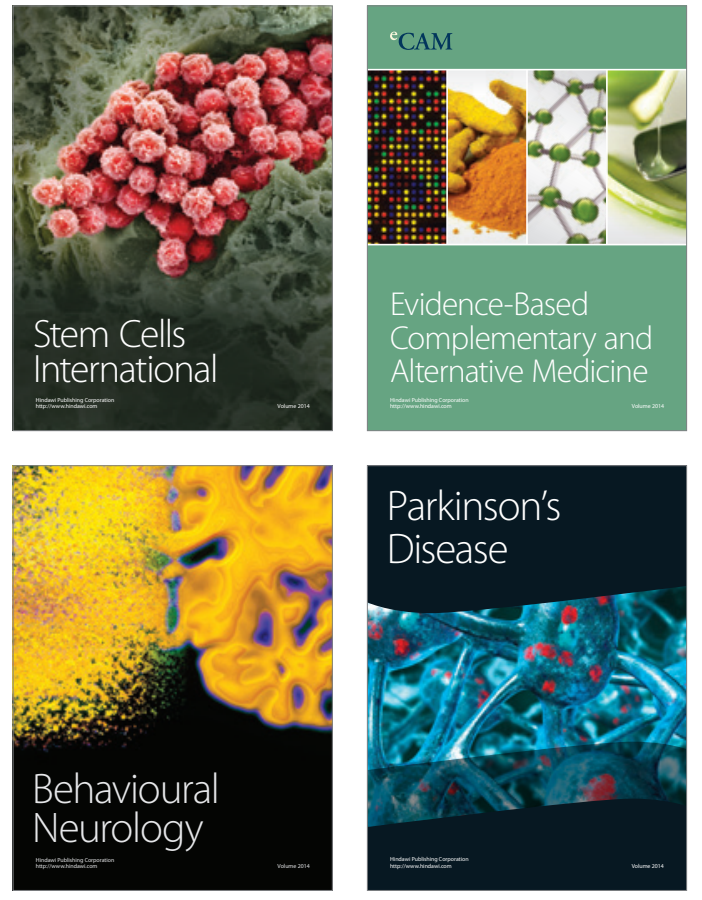
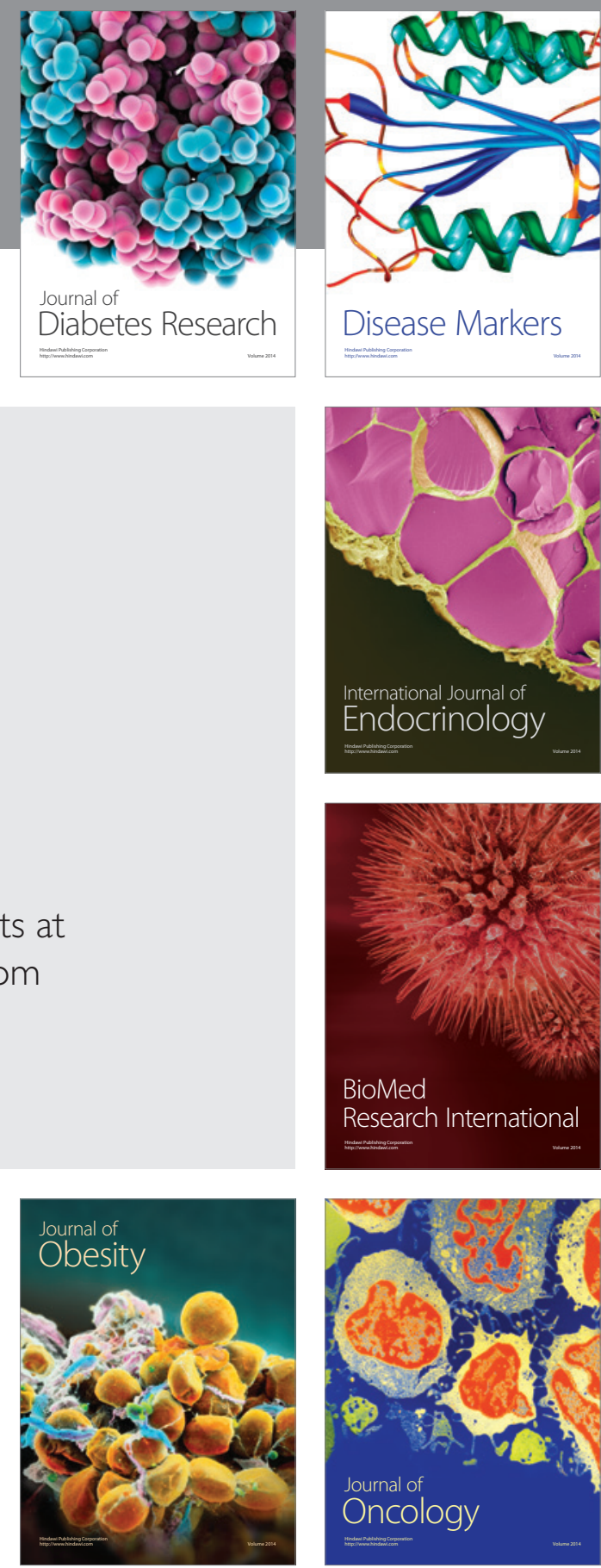

Disease Markers
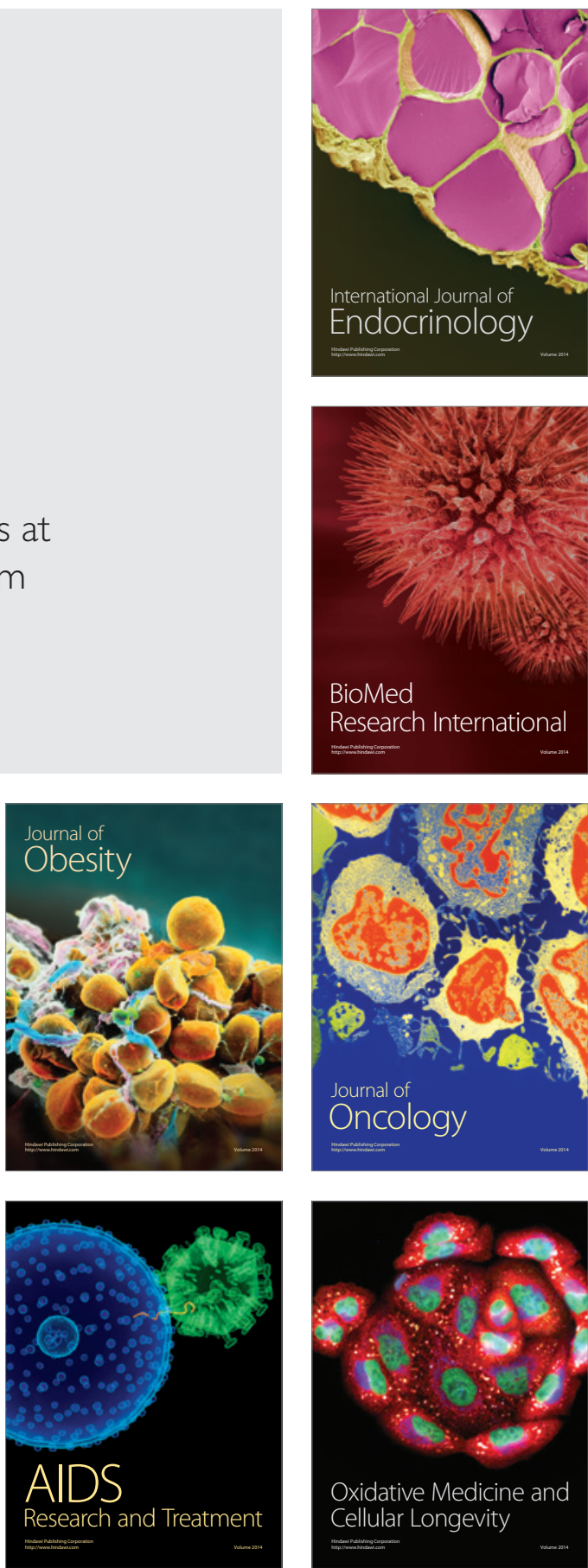